\title{
Efficient Frequent Connected Subgraph Mining in Graphs of Bounded Treewidth
}

\author{
Tamás Horváth ${ }^{1,2}$ and Jan Ramon ${ }^{3}$ \\ ${ }^{1}$ Dept. of Computer Science III, University of Bonn, Germany \\ ${ }^{2}$ Fraunhofer IAIS, Schloss Birlinghoven, Sankt Augustin, Germany \\ tamas.horvath@iais.fraunhofer.de \\ ${ }^{3}$ Dept. of Computer Science, Katholieke Universiteit Leuven, Belgium \\ jan.ramon@cs. kuleuven. be
}

\begin{abstract}
The frequent connected subgraph mining problem, i.e., the problem of listing all connected graphs that are subgraph isomorphic to at least a certain number of transaction graphs of a database, cannot be solved in output polynomial time in the general case. If, however, the transaction graphs are restricted to forests then the problem becomes tractable. In this paper we generalize the positive result on forests to graphs of bounded treewidth. In particular, we show that for this class of transaction graphs, frequent connected subgraphs can be listed in incremental polynomial time. Since subgraph isomorphism remains NPcomplete for bounded treewidth graphs, the positive complexity result of this paper shows that efficient frequent pattern mining is possible even for computationally hard pattern matching operators.
\end{abstract}

\section{Introduction}

During the last decade, graph mining developed into a separate field of knowledge discovery in databases, motivated by various practical applications for example in bioinformatics, computational chemistry, and the WWW. A basic task in this field is the frequent connected subgraph mining (FCSM) problem: Given a database of labeled graphs, called transaction graphs, and some positive integer threshold $t$, list all connected graphs that are subgraph isomorphic to at least $t$ transaction graphs. Such frequent connected patterns have successfully been used, for example, in ligand-based virtual screening as features 6 .

For arbitrary transaction graphs, the FCSM problem cannot be solved in output-polynomial time (if $\mathrm{P} \neq \mathrm{NP}$ ) [10]. While several heuristic methods have been developed for this general problem that proved to be effective on various graph datasets, surprisingly there are only few results about tractable graph classes. To the best of our knowledge, the only positive (non-trivial) result towards this direction is about forests; the FCSM problem can be solved in incremental polynomial time for forest transaction graphs (see [5] for a survey on tree mining). The exploration of the border between tractable and intractable graph classes is an important theoretical challenge because it could provide useful insights into the problem which could then be exploited in the design of practical algorithms. 
In this paper we take a step towards this goal by generalizing the positive result on forests to graphs of bounded treewidth. Treewidth [14 is a measure of tree-likeness of graphs that proved to be a useful property in algorithmic graph theory because several NP-hard problems on graphs become tractable for the class of bounded treewidth graphs. This class is also of practical importance, as it includes many graph classes appearing in practical applications (see, e.g., 24]). For example, the molecular graphs of the vast majority of pharmacological compounds have treewidth at most 3 .

We present a levelwise search algorithm listing frequent connected subgraphs in incremental polynomial time if the treewidth of the transaction graphs is bounded by some constant. We make use of the fact that isomorphism between graphs of bounded treewidth can be decided efficiently 2 . To calculate the support count of candidate patterns, we use a modification of the subgraph isomorphism algorithm developed for graphs of bounded treewidth and log-bounded fragmentation [9], where the class of log-bounded fragmentation graphs properly contains the class of bounded degree graphs. This algorithm is based on a fundamental generic algorithm designed for deciding various morphisms between graphs of bounded treewidth and bounded degree [13. In a nutshell, the main result of 13 is that several graph morphisms, including subgraph isomorphism, can be decided efficiently by a dynamic programming algorithm computing polynomially many, polynomial time computable properties if the treewidth and the degree of the graphs are both bounded by some constant.

Since we do not assume any bound on the degree, the number of such properties can be exponentially large. We can show, however, that for a given candidate pattern $H$, it is sufficient to compute only a polynomially large subset of these properties; the rest, maybe exponentially large set, can be derived from those of the frequent subgraphs listed before $H$. To show this result, we utilize the levelwise generation of frequent patterns and the anti-monotonic property of frequency. In this way, the delay can be exponential in the size of the input only after the enumeration of exponentially many frequent patterns. This technique might be of some independent interest and useful to design efficient algorithms where straightforward dynamic programming would require exponential space.

We note that subgraph isomorphism remains NP-complete even for connected graphs of bounded treewidth (see, e.g., [13]). The positive result of this paper thus provides an example of the case when efficient frequent pattern mining is possible even for NP-hard pattern matching operators. A significant consequence of our result is thus immediate to the study of frequent pattern mining: Efficient frequent pattern mining is possible even for NP-hard pattern matching operators.

The rest of the paper is organised as follows. In Section 2 we first collect the necessary notions and fix the notations. In Section 3 we present a generic levelwise frequent connected subgraph mining algorithm and analyse its computational properties. In Section 4 we adapt this generic algorithm to graphs of bounded treewidth and show that it lists frequent connected subgraphs in incremental polynomial time. Finally, in Section [5] we conclude along with an open problem. Due to space limitations, proofs are omitted in this short version. 


\section{Preliminaries}

In this section we first briefly review some basic concepts and fix the notations used in this paper. We start with some standard definitions from graph theory.

Graphs. An undirected graph is a pair $(V, E)$, where $V$ is a finite set of vertices and $E \subseteq\{e \subseteq V:|e|=2\}$ is a set of edges. A labeled undirected graph is a triple $(V, E, \lambda)$, where $(V, E)$ is an undirected graph and $\lambda: V \cup E \rightarrow \mathbb{N}$ is a function assigning a label to every element of $V \cup E$. Unless otherwise stated, in this paper by graphs we always mean labeled undirected graphs and denote the set of vertices, the set of edges, and the labeling function of a graph $G$ by $V(G)$, $E(G)$, and $\lambda_{G}$, respectively.

Let $G$ and $G^{\prime}$ be graphs. Then $G^{\prime}$ is a subgraph of $G$, if $V\left(G^{\prime}\right) \subseteq V(G)$, $E\left(G^{\prime}\right) \subseteq E(G)$, and $\lambda_{G^{\prime}}(x)=\lambda_{G}(x)$ for every $x \in V\left(G^{\prime}\right) \cup E\left(G^{\prime}\right)$; it is an induced subgraph of $G$ if it is a subgraph of $G$ satisfying $\{u, v\} \in E\left(G^{\prime}\right)$ iff $\{u, v\} \in E(G)$ for every $u, v \in V\left(G^{\prime}\right)$. For a subset $S \subseteq V(G), G[S]$ denotes the induced subgraph of $G$ with vertex set $S$.

A path connecting the vertices $v_{1}, v_{k} \in V(G)$ in a graph $G$ is a sequence $\left\{v_{1}, v_{2}\right\},\left\{v_{2}, v_{3}\right\}, \ldots,\left\{v_{k-1}, v_{k}\right\} \in E(G)$ such that the $v_{i}$ 's are pairwise distinct. A graph is connected if there is a path between any pair of its vertices. A connected component of a graph $G$ is a maximal subgraph of $G$ that is connected. The set of all connected components of a graph $G$ is denoted by $\mathcal{C}(G)$.

Isomorphism and Subgraph Isomorphism. Let $G_{1}$ and $G_{2}$ be graphs. They are isomorphic if there is a bijection $\varphi: V\left(G_{1}\right) \rightarrow V\left(G_{2}\right)$ satisfying (i) $\{u, v\} \in$ $E\left(G_{1}\right)$ iff $\{\varphi(u), \varphi(v)\} \in E\left(G_{2}\right)$ for every $u, v \in V\left(G_{1}\right)$, (ii) $\lambda_{G_{1}}(u)=\lambda_{G_{2}}(\varphi(u))$ for every $u \in V\left(G_{1}\right)$, and (iii) $\lambda_{G_{1}}(\{u, v\})=\lambda_{G_{2}}(\{\varphi(u), \varphi(v)\})$ for every $\{u, v\} \in$ $E\left(G_{1}\right)$. In this paper, isomorphic graphs are regarded as identical graphs.

For $G_{1}$ and $G_{2}$ above we say that $G_{1}$ is subgraph isomorphic to $G_{2}$, denoted $G_{1} \leq G_{2}$, if $G_{1}$ is isomorphic to a subgraph of $G_{2}$. Deciding whether a graph is subgraph isomorphic to another graph is NP-complete, as it generalizes, e.g., the Hamiltonian path problem 7 ].

Treewidth. The notion of treewidth was reintroduced in 14. It proved to be a useful parameter of graphs in algorithmic graph theory. A tree-decomposition of a graph $G$, denoted $T D(G)$, is a pair $(T, \mathcal{X})$, where $T$ is an unordered tree and $\mathcal{X}=\left(X_{z}\right)_{z \in V(T)}$ is a family of subsets of $V(G)$ satisfying (i) $\cup_{z \in V(T)} X_{z}=V(G)$, (ii) for every $\{u, v\} \in E(G)$, there is a $z \in V(T)$ such that $u, v \in X_{z}$, and (iii) $X_{z_{1}} \cap X_{z_{3}} \subseteq X_{z_{2}}$ for every $z_{1}, z_{2}, z_{3} \in V(T)$ such that $z_{2}$ is on the path connecting $z_{1}$ with $z_{3}$ in $T$. The set $X_{z}$ associated with a node $z$ of $T$ is called the bag of $z$. The nodes of $T$ will often be referred to as the nodes of $T D(G)$. The treewidth of $T D(G)$ is $\max _{z \in V(T)}\left|X_{z}\right|-1$, and the treewidth of $G$, denoted tw $(G)$, is the minimum treewidth over all tree-decompositions of $G$. By graphs of bounded treewidth we mean graphs of treewidth at most $k$, where $k$ is some constant.

The following notation will be used many times in what follows. Let $G$ be a graph, $T D(G)=(T, \mathcal{X})$ be a tree-decomposition of $G$, and $z \in V(T)$. Then $G_{[z]}$ denotes the induced subgraph of $G$ defined by the union of the bags of $z$ 's descendants. (We note that $z$ is considered also as a descendant of itself.) 


\section{Mining Frequent Connected Subgraphs}

In this section we first define the frequent connected subgraph mining problem, present a generic listing algorithm based on levelwise search for this problem, and provide sufficient conditions for the efficiency of this algorithm. Applying these conditions, we then show that the frequent connected subgraph mining problem can be solved in incremental polynomial time for forest transaction graphs and for transaction graphs that have bounded treewidth and log-bounded fragmentation. In the next section we will show how to adapt the generic algorithm of this section to mining frequent connected subgraphs from graphs of bounded treewidth that do not necessarily have log-bounded fragmentation. We start with the definition of the general problem setting.

The Frequent Connected Subgraph Mining (FCSM) Problem: Given a class $\mathcal{G}$ of graphs, a transaction database $D B$ of graphs from $\mathcal{G}$ (i.e., a multiset of graphs from $\mathcal{G}$ ), and an integer threshold $t>0$, list the set of frequent connected subgraphs, that is, the set of connected graphs that are subgraph isomorphic to at least $t$ graphs in $D B$.

The parameter of the above problem is the size of $D B$. One can easily construct examples when the number of frequent connected subgraphs is exponential in this parameter. Thus, in general, the set of all frequent connected subgraphs cannot be computed in time polynomial in the size of $D B$. Since this is a common feature of listing problems, the following problem classes are usually considered in the literature (see, e.g., [12]): Let $S$ be a set of cardinality $N$. Then its elements, say $s_{1}, \ldots, s_{N}$, are listed with

polynomial delay if the time before printing $s_{1}$, the time between printing $s_{i}$ and $s_{i+1}$ for every $i=1, \ldots, N-1$, and the termination time after printing $s_{N}$ is bounded by a polynomial of the size of the input,

incremental polynomial time if $s_{1}$ is printed with polynomial delay, the time between printing $s_{i}$ and $s_{i+1}$ for every $i=1, \ldots, N-1$ (resp. the termination time after printing $s_{N}$ ) is bounded by a polynomial of the combined size of the input and the set $\left\{s_{1}, \ldots, s_{i}\right\}$ (resp. $S$ ),

output polynomial time (or polynomial total time) if $S$ is printed in the combined size of the input and the entire set $S$.

Clearly, polynomial delay implies incremental polynomial time, which, in turn, implies output polynomial time. Furthermore, in contrast to incremental polynomial time, the delay of an output polynomial time algorithm may be exponential in the size of the input even before printing the first element of the output.

Although several frequent connected subgraph mining algorithms have been developed that proved to be effective in various practical applications, we note that, unless $\mathrm{P}=\mathrm{NP}$, the FCSM problem cannot be solved in output polynomial time for arbitrary transaction graphs 10. It can be solved, however, in incremental polynomial time if the transaction graphs are restricted to forests (see, e.g., 5[11). The main contribution of this work is to extend this positive result to graphs of bounded treewidth. To achieve this goal, we first give a generic levelwise mining algorithm designed for downward closed graph classes. 


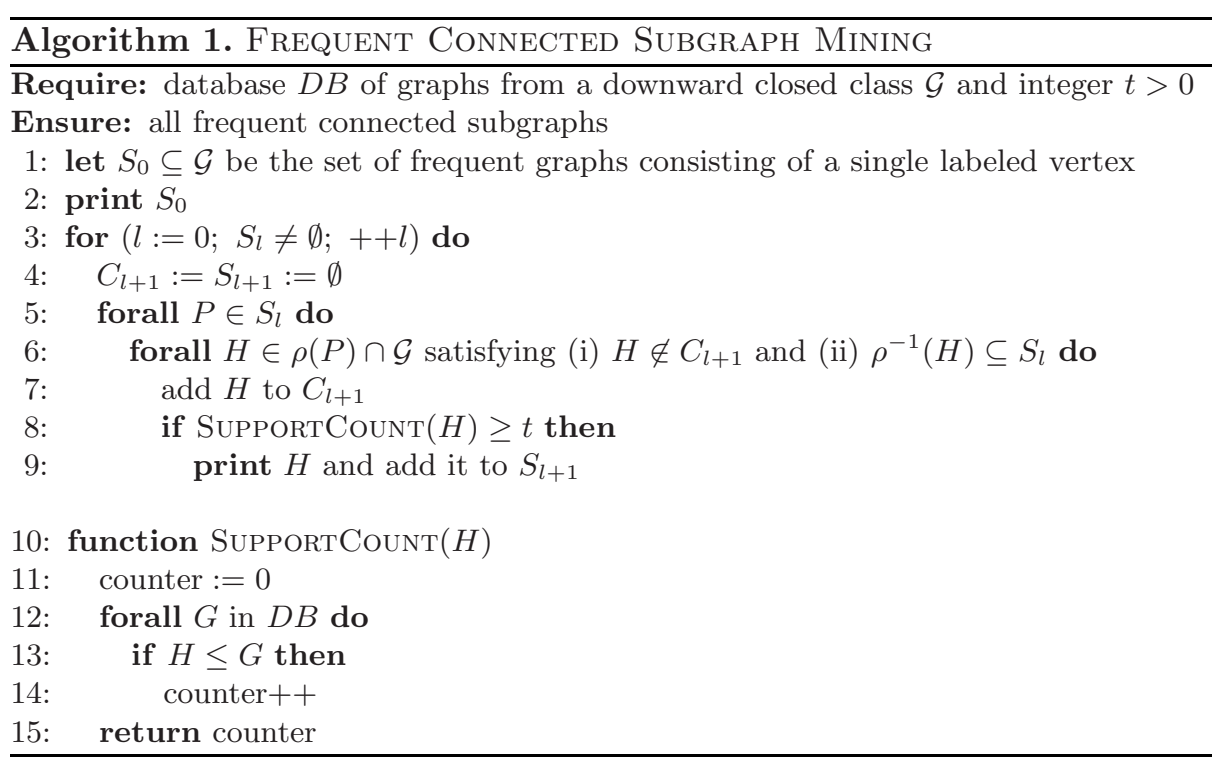

\subsection{A Generic Mining Algorithm}

A generic algorithm listing frequent connected subgraphs with levelwise search is given in Algorithm 11. It requires the class $\mathcal{G}$ of transaction graphs to be closed downward, i.e., for every $G \in \mathcal{G}, \mathcal{G}$ contains all subgraphs of $G$. In the algorithm we first compute the set of frequent graphs from $\mathcal{G}$ that consist of a single vertex. (We recall that by graphs we mean labeled graphs.) In the main loop we then iteratively compute the set $S_{l+1}$ of frequent connected graphs containing $l+1$ edges from those containing $l$ edges for every $l \geq 0$. In particular, for each frequent pattern $P \in S_{l}$, we compute the set $\rho(P) \cap \mathcal{G}$ of graphs, where the elements of $\rho(P)$ are obtained from $P$ by either connecting two vertices with a labeled edge or by adding a new labeled vertex to $P$ and connecting it with an old vertex by a labeled edge. Clearly, the graphs in $\rho(P)$ are all connected, as $P$ is connected. For each $H \in \rho(P) \cap \mathcal{G}$, we check whether or not it has already been generated during the current iteration step of the main loop (condition (i) in line 6). If not, we also check for each graph in $\rho^{-1}(H)$ whether it is frequent, where $\rho^{-1}(H)$ is the set of connected graphs obtained from $H$ by removing an edge (condition (ii) in line 6). Throughout this paper, candidate patterns generated by levelwise search that satisfy conditions (i) and (ii) in line 6 are called strong candidates. If $H$ is a strong candidate, we add it to the set $C_{l+1}$ of candidate graphs consisting of $l+1$ edges and compute its support count. If it is frequent, i.e., subgraph isomorphic to at least $t$ graphs in $D B$, we print it and add it to the set $S_{l+1}$ of frequent connected graphs containing $l+1$ edges.

As mentioned earlier, without any further assumption on $\mathcal{G}$, the FCSM problem cannot be solved in output polynomial time [10]. If, however, $\mathcal{G}$ satisfies the conditions of the theorem below, one can obtain tractable problem classes. 
Theorem 1. Let $\mathcal{G}$ be a graph class satisfying the following properties:

(i) $\mathcal{G}$ is closed downward,

(ii) the membership problem in $\mathcal{G}$, i.e., whether $G \in \mathcal{G}$ for any graph $G$ can be decided in polynomial time, and

(iii) for every $H, G \in \mathcal{G}$ such that $H$ is connected, it can be decided in polynomial time whether $H$ is subgraph isomorphic to $G$ (i.e., if $H \leq G$ ).

If the transaction graphs in $D B$ belong to $\mathcal{G}$ then Algorithm 1 lists the frequent connected subgraphs in incremental polynomial time.

Notice that condition (i) above does not require the elements of $\mathcal{G}$ to be connected. The proof of the theorem is straightforward by noting that the cardinality and hence, the sizes of the sets $\rho(H) \cap \mathcal{G}$ and $\rho^{-1}(H)$ in line 6 are bounded by a polynomial of the size of $D B$, and both sets can be computed in polynomial time. Thus, the size of $C_{l+1}$ is bounded by the combined size of $D B$ and $S_{l}$.

Without proof, we now mention two immediate applications of the above theorem. The first result on forests follows from several results (see, e.g., [511]).

Corollary 1. The FCSM problem can be solved in incremental polynomial time for forest transaction graphs.

To state a second application of Theorem 1] we first need a definition. A graph $G$ has $k$-log-bounded (or simply, log-bounded) fragmentation 9] if

$$
|\mathcal{C}(G[V(G) \backslash S])|-|\mathcal{C}(G)|=O(k \log |V(G)|)
$$

for every $S \subseteq V(G)$ satisfying $|S| \leq k$. In other words, $G$ has log-bounded fragmentation if the removal of any set of at most $k$ vertices and all adjacent edges from $G$ results in a graph with $|\mathcal{C}(G)|+O(k \log |V(G)|)$ connected components. As an obvious example, graphs with maximum degree bounded by some constant have log-bounded fragmentation. Using Theorem 1 for graphs of bounded treewidth and log-bounded fragmentation, the following result can be shown:

Corollary 2. The FCSM problem can be solved in incremental polynomial time if the transaction graphs have bounded treewidth and log-bounded fragmentation.

Consider the NCI chemical databast 1 used as a benchmark graph dataset in graph mining. Out of the 250251 molecular graphs in this dataset, 243638 (i.e., $97,36 \%$ ) compounds have treewidth at most 2, 250186 (i.e., 99,97\%) treewidth at most 3 ; there are only 65 (i.e., $0,03 \%$ ) compounds with treewidth at least 4 . However, compounds of small treewidth have often relatively large degree.

Motivated by applications of frequent connected subgraphs in computational drug design, in particular, in ligand-based virtual screening (see, e.g., 6]), it is therefore natural to ask if the positive result of Corollary 2 can be extended to arbitrary graphs of bounded treewidth. This raises the question whether condition (iii) of Theorem 1 is indeed necessary because subgraph isomorphism between graphs of bounded treewidth is NP-complete even for connected patterns (see, e.g., [13]). In the rest of this paper we show that for graphs of bounded treewidth, condition (iii) is unnecessary.

1 http://cactus.nci.nih.gov/ 


\section{Mining Frequent Connected Subgraphs in Graphs of Bounded Treewidth}

Consider again Theorem 1 It is easy to see that condition (i) holds for arbitrary graphs of bounded treewidth. Regarding condition (ii), for any constant $k$, one can decide in linear time whether a graph has treewidth at most $k$ and if yes, compute a tree-decomposition of treewidth at most $k[3$. However, condition (iii) on the efficiency of subgraph isomorphism does not hold for arbitrary connected graphs of bounded treewidth. For graphs of bounded treewidth, this condition has a side effect only in the support counting step (line 8) which is based on deciding subgraph isomorphism (line 13). Thus, to show that the FCSM problem can be solved in incremental polynomial time for graphs of bounded treewidth, it is sufficient to show that subgraph isomorphism can be decided in time polynomial in the combined size of the input and the set of frequent patterns computed before the current candidate pattern.

The subgraph isomorphism problem remains NP-complete even when both the pattern and transaction graphs have bounded treewidth and the pattern graph is connected [13. The NP-completeness of the subforest isomorphism problem [7] implies that the connectivity of the pattern graph is necessary even for acyclic graphs. For graphs of treewidth at most $k$, there is a clear demarcation between tractable and intractable instances of the subgraph isomorphism problem: if the pattern is not $k$-connected or has more than $k$ vertices of unbounded degree then the subgraph isomorphism is NP-complete; otherwise it can be decided in polynomial time [8]. However, as we show below, for efficient mining of frequent connected subgraphs, these conditions are unnecessary because the antimonotonicity of frequency allows the mining algorithm to utilise the information computed earlier for the candidates' frequent ancestors.

The rest of this section is organised as follows. In Section 4.1 we first overview the subgraph isomorphism algorithm described in [9]. In Section 4.2 we then state, without proof, that for every connected graphs $H$ and $G$ of bounded treewidth, this algorithm computes only a polynomial number of new properties needed to decide $H \leq G$; any property from the rest, possibly exponentially many properties, can be derived from the properties computed for the frequent patterns in the previous steps. Finally, in Section 4.3, we show how to integrate this modified subgraph isomorphism algorithm into Algorithm 1.

\subsection{Subgraph Isomorphism between Graphs of Bounded Treewidth and Log-Bounded Fragmentation}

Throughout this section $H$ and $G$ denote connected graphs of treewidth at most $k$, where $k$ is some constant. In fact, we need only $H$ to be connected. Since any subgraph isomorphism maps a connected graph into a connected component of a graph, we may assume without loss of generality that $G$ is also connected.

Following the dynamic-programming approach of [1, the algorithm described in 9] first computes a nice tree-decomposition $T D(G)=(T, \mathcal{X})$ of $G$, where a 
nice tree-decomposition is a tree-decomposition such that $T$ is a rooted binary tree composed of three types of nodes: (i) a leaf node has no children, (ii) a separator node $z$ has a single child $z^{\prime}$ with $X_{z} \subseteq X_{z^{\prime}}$, and (iii) a join node $z$ has two children $z_{1}$ and $z_{2}$ with $X_{z}=X_{z_{1}} \cup X_{z_{2}}$. It follows from the results in [4] that for graphs of treewidth at most $k$, such a nice tree-decomposition of treewidth at most $k$ always exists and can be constructed in linear time.

Given a nice tree-decomposition $T D(G)$ of $G$, the algorithm in 9] computes a set of tuples (properties) for each node in a bottom-up manner and decides whether $H \leq G$ holds by checking a condition for the set computed for the root of $T D(G)$. To define this condition precisely, we need some notions. For a node $z$ in $T D(G)$, a partial solution relative to $z$ is a subgraph isomorphism from a subgraph $H^{\prime}$ of $H$ into $G_{[z]}$. An iso-quadruple relative to a node $z$ of $T D(G)$ is a quadruple $(S, \mathcal{D}, K, \psi)$, where

$-S \subseteq V(H)$ satisfying $|S| \leq k+1$,

$-\mathcal{D} \subseteq \mathcal{C}(H[V(H) \backslash S])$,

- $K=H[S \cup V(\mathcal{D})]$, and

$-\psi$ is an injective function mapping $S$ to $X_{z}$.

We note that $K$ is redundant in the above notation; we add it to the tuple only for the reader's convenience. The set of all iso-quadruples of $H$ relative to a node $z$ of $T D(G)$ is denoted by $\Gamma(H, z)$.

We need some further definitions. A characteristic of a partial solution $\varphi$ relative to a node $z$ in $T D(G)$ is an iso-quadruple $(S, \mathcal{D}, K, \psi) \in \Gamma(H, z)$ such that $\varphi$ is a subgraph isomorphism from $K$ to $G_{[z]}$ satisfying $\varphi(u)=\psi(u)$ for every $u \in S$ and $\varphi(v) \notin X_{z}$ for every $v \in V(\mathcal{D})$. The definitions imply that $\varphi(u) \in X_{z}$ for every $u \in S$. Finally, a $z$-characteristic is an iso-quadruple in $\Gamma(H, z)$ that is the characteristic of at least one partial solution relative to $z$. The set of all $z$-characteristics of $H$ relative to $z$ is denoted by $\Gamma_{\operatorname{ch}}(H, z)$. Clearly, $\Gamma_{\mathrm{ch}}(H, z) \subseteq \Gamma(H, z)$. The following lemma from [9] provides a characterization of subgraph isomorphism in terms of $r$-characteristics for the root $r$ of $T D(G)$ :

Lemma 1. Let $r$ be the root of a nice tree-decomposition $T D(G)$ of $G$. Then $H \leq G$ iff there exists an iso-quadruple $(S, \mathcal{D}, H, \psi)$ in $\Gamma_{\mathrm{ch}}(H, r)$.

Notice that by definition, $S$ can be the empty set. Thus, by the above lemma, we can decide $H \leq G$ by computing and testing the set of $r$-characteristics for the root $r$ of $T D(G)$. This can be carried out by traversing $T D(G)$ in a postorder manner and computing the set of characteristics for each non-leaf node from those of its children. Depending on the type of the current node $z$ visited, we perform one of the following steps (for more details on the method described below, the reader is referred, e.g., to [9]):

Leaf Nodes: If $z$ is a leaf in $T D(G)$ then for all $(S, \mathcal{D}, K, \psi) \in \Gamma_{\operatorname{ch}}(H, z)$ it holds that $\mathcal{D}=\emptyset$ and $K=H[S]$. For this case, the following lemma holds.

Lemma 2. Let $z$ be a leaf in $T D(G)$ and $\xi=(S, \emptyset, H[S], \psi) \in \Gamma(H, z)$. Then $\xi \in \Gamma_{\mathrm{ch}}(H, z)$ iff $\psi$ is a subgraph isomorphism from $H[S]$ to $G\left[X_{z}\right]$. 
Since $S$ and $X_{z}$ both have at most $k+1$ vertices, $|\Gamma(H, z)|$ is bounded by $(k+1) ! \cdot|V(H)|^{k+1}$. Furthermore, using the above lemma, one can decide in time $(k+1)^{2}$, whether an iso-quadruple in $\Gamma(H, z)$ is a $z$-characteristic. Thus, the set of characteristics for leaf nodes can be computed in polynomial time.

Separator Nodes: If $z$ is a separator node then, by definition, it has a single child $z^{\prime}$ with $X_{z} \subseteq X_{z^{\prime}}$. For an iso-quadruple $\xi=(S, \mathcal{D}, K, \psi) \in \Gamma(H, z)$, let $\Pi(\xi)$ denote the set of iso-quadruples $\left(S^{\prime}, \mathcal{D}^{\prime}, K^{\prime}, \psi^{\prime}\right) \in \Gamma\left(H, z^{\prime}\right)$ satisfying

(i) $S=\left\{v \in S^{\prime}: \psi^{\prime}(v) \in X_{z}\right\}$,

(ii) $\mathcal{D}^{\prime}=\left\{D^{\prime} \in \mathcal{C}\left(H\left[V(H) \backslash S^{\prime}\right]\right): D^{\prime}\right.$ is a subgraph of some $\left.D \in \mathcal{D}\right\}$, and

(iii) $\psi(v)=\psi^{\prime}(v)$ for every $v \in S$.

Using this definition, the set of characteristics relative to a separator node can be computed by the following lemma.

Lemma 3. Let $z$ be a separator node in $T D(G)$ with child $z^{\prime}$ and $\xi \in \Gamma(H, z)$ be an iso-quadruple. Then $\xi \in \Gamma_{\mathrm{ch}}(H, z)$ iff $\Gamma_{\mathrm{ch}}\left(H, z^{\prime}\right) \cap \Pi(\xi) \neq \emptyset$.

For a separator node $z$ with child $z^{\prime}$ and $\xi \in \Gamma(H, z),|\Pi(\xi)|$ is bounded by $(k+1) ! \cdot|V(H)|^{k+1}$. Using some advanced data structure for storing the set of characteristics of a node, one can decide in polynomial time whether an iso-quadruple $\xi^{\prime} \in \Pi(\xi)$ is a $z^{\prime}$-characteristic. Thus, for an iso-quadruple $\xi$ in $\Gamma(H, z)$, we can decide in polynomial time whether $\xi$ is a $z$-characteristic if the set of $z^{\prime}$-characteristics has already been computed. However, $|\Gamma(H, z)|$ can be exponential in the size of $H$ if only its treewidth is restricted.

Join Nodes: If $z$ is a join node then it has two children $z_{1}$ and $z_{2}$ with bags satisfying $X_{z}=X_{z_{1}} \cup X_{z_{2}}$. From the sets of characteristics relative to the children of $z$, the set of $z$-characteristics can be computed by using the following lemma:

Lemma 4. Let $z$ be a join node in $T D(G)$ with children $z_{1}$ and $z_{2}$, and let $\xi=(S, \mathcal{D}, K, \psi) \in \Gamma(H, z)$ be an iso-quadruple. Then $\xi \in \Gamma_{\mathrm{ch}}(H, z)$ iff there exist $\left(S_{1}, \mathcal{D}_{1}, K_{1}, \psi_{1}\right) \in \Gamma_{\mathrm{ch}}\left(H, z_{1}\right)$ and $\left(S_{2}, \mathcal{D}_{2}, K_{2}, \psi_{2}\right) \in \Gamma_{\mathrm{ch}}\left(H, z_{2}\right)$ such that

(i) $S_{i}=\left\{v \in S: \psi(v) \in X_{z_{i}}\right\}$ for $i=1,2$,

(ii) the connected components of $\mathcal{D}$ are partitioned into $\mathcal{D}_{1}$ and $\mathcal{D}_{2}$,

(iii) for $i=1,2, \psi_{i}(v)=\psi(v)$ for every $v \in S_{i}$, and

(iv) $\psi$ preserves the labels and the edges.

By condition (ii) above we have to take the set of all possible partitionings of a set $\mathcal{D}$ of connected components, which is exponential in the number of connected components of $\mathcal{D}$. Thus, the situation is much worse for join nodes than for separator nodes because not only the number of $z$-characteristics can be exponential, but the computation of a particular $z$-characteristic may involve exponentially many tests.

In case of log-bounded fragmentation graphs, the set of characteristics can be computed in polynomial time for separator and join nodes as well 9. If, however, we do not assume any further restrictions on connected graphs of bounded treewidth, the above bottom-up evaluation may require exponential time. 


\subsection{Subgraph Isomorphism between Graphs of Bounded Treewidth}

In this section we show that the subgraph isomorphism algorithm described above can be integrated into Algorithm 1 in such a way that for any strong candidate pattern $H$ (i.e., which satisfies conditions (i) and (ii) in line 6 of Algorithm 10 and for any transaction graph $G \in D B, H \leq G$ can be decided in time polynomial in the combined size of $H, G$, and the set of frequent patterns listed before $H$. The key observation is that it is sufficient to compute only polynomially many characteristics for $H$ and to use the characteristics of the frequent patterns computed before $H$. We recall that Algorithm 1 lists the set of frequent patterns with levelwise search. Thus, all connected subgraphs $H^{\prime}$ of $H$ have already been processed in the frequency counting step. One of the changes in the algorithm is that for every transaction graph $G$, we compute a nice tree-decomposition $T D(G)$ in a preprocessing step and use this decomposition during the entire mining process. This is necessary for the reutilisation of the characteristics computed for the frequent patterns in earlier steps.

Non-Redundant Iso-Quadruples. We start with a definition that will be used to determine the set of characteristics of a strong candidate pattern $H$ relative to a node, which cannot be recovered from the set of characteristics of the frequent patterns computed before $H$. Let $H_{1}, H_{2}$, and $G$ be connected graphs of bounded treewidth, $T D(G)$ be the nice tree-decomposition of $G$ computed in the preprocessing step, and $z$ be a node in $T D(G)$. Let $\xi_{1}=\left(S_{1}, \mathcal{D}_{1}, K_{1}, \psi_{1}\right) \in$ $\Gamma\left(H_{1}, z\right)$ and $\xi_{2}=\left(S_{2}, \mathcal{D}_{2}, K_{2}, \psi_{2}\right) \in \Gamma\left(H_{2}, z\right)$ be iso-quadruples relative to $z$. We say that $\xi_{1}$ is equivalent to $\xi_{2}$, denoted $\xi_{1} \equiv \xi_{2}$, if there is an isomorphism $\pi$ between $K_{1}$ and $K_{2}$ such that $\pi$ is a bijection between $S_{1}$ and $S_{2}$ and $\psi_{1}(v)=$ $\psi_{2}(\pi(v))$ for every $v \in S_{1}$. The proof of the proposition below follows directly from the definitions.

Proposition 1. Let $\xi_{1}$ and $\xi_{2}$ be equivalent iso-quadruples relative to a node $z$ of $T D(G)$. Then $\xi_{1}$ is a $z$-characteristic iff $\xi_{2}$ is a $z$-characteristic.

In order to utilise the information computed previously, for each node $z$ in $T D(G)$, we store the set of $z$-characteristics computed for the frequent patterns listed earlier by the algorithm. Proposition 1 above implies that it is sufficient to store only one representative $z$-characteristic from each equivalence class of the set of $z$-characteristics. The following definition will be used many times in what follows. An iso-quadruple $\xi \in \Gamma(H, z)$ of a strong candidate pattern $H$ is redundant if there is an equivalent iso-quadruple in $\Gamma(P, z)$ for some frequent pattern $P$ computed before $H$. The set of non-redundant iso-quadruples of a pattern $H$ relative to a node $z$ in $T D(G)$ is denoted by $\Gamma_{\mathrm{nr}}(H, z)$.

For a strong candidate pattern $H$ and iso-quadruple $\xi \in \Gamma(H, z)$, we have to test whether $\xi$ is a $z$-characteristic of $T D(G)$ only when $\xi$ is non-redundant; if $\xi$ is non-redundant, we add it to the set of characteristics stored for $z$ only if it is a $z$-characteristic. The number of iso-quadruples of a pattern relative to a node $z$ can be exponential. Theorem 2 below is of special importance for the main result of this paper, as it implies that the number of non-redundant iso-quadruples of 
a strong candidate pattern is always bounded by a polynomial of the combined size of the pattern and the largest graph in the transaction database. In the following lemma we give an upper bound on the number of non-redundant isoquadruples of a pattern relative to a node. Due to space limitations, we omit the proof which is based on combinatorial arguments.

Lemma 5. Let $H$ be a strong candidate pattern generated by levelwise search and let $z$ be a node in $T D(G)$ for some transaction graph $G$. Then $\left|\Gamma_{\mathrm{nr}}(H, z)\right|$ is bounded by $O\left(|V(H)|^{k+1}\right)$.

Using the above lemma, we can state the following result concerning nonredundant iso-quadruples of a pattern.

Theorem 2. In order to decide $H \leq G$, it is sufficient to check for at most $O\left(|V(G)| \cdot|V(H)|^{k+1}\right)$ iso-quadruples of $H$, whether they are characteristics relative to some node of $T D(G)$.

Proof. To decide $H \leq G$, it is sufficient to check non-redundant iso-quadruples. The statement then follows directly from Lemma 5 by noting that the size of $T D(G)$ is linear in that of $G[3]$.

By Lemmas 3 and 4 . for a node $z$ in $T D(G)$, the set of $z$-characteristics is computed from the sets of $z^{\prime}$-characteristics of the children $z^{\prime}$ of $z$. Looking up from the set of $z^{\prime}$-characteristics, one can decide in time polynomial in the size of $H$, whether an iso-quadruple $\xi=(S, \mathcal{D}, K, \psi)$ is a $z^{\prime}$-characteristic. We omit the details of this technical result from this short version. The key is that we color each vertex $u \in S$ of $K$ by $\psi(u) \in X_{z^{\prime}}$ and compute a canonical string representation of this colored graph obtained from $K$. This canonical string representation is unique modulo isomorphism and can be computed in time polynomial in the size of $K$. Storing the canonical string representation of the $z^{\prime}$-characteristics in some advanced data structure, e.g., in prefix trees, we can decide in linear time, whether there exists a characteristic equivalent to $\xi$ among the set of $z^{\prime}$-characteristics computed so far for the frequent patterns.

Deciding Subgraph Isomorphism. We now turn to the question of how to decide subgraph isomorphism using only non-redundant characteristics. Let $H$ be a strong candidate pattern generated by levelwise search. For a transaction graph $G$ and node $z$ in $T D(G)$, let $\Sigma(z)$ and $\Gamma_{\mathrm{nr}, \mathrm{ch}}(H, z)$ denote the set of all non-redundant $z$-characteristics computed for the frequent patterns listed before $H$ and the set of non-redundant $z$-characteristics computed for $H$, respectively. Let $r$ be the root of $T D(G)$. Then Lemma 1 and Proposition 1 together imply that $H \leq G$ if and only if there are iso-quadruples $\xi=(S, \mathcal{D}, H, \psi) \in \Gamma(H, r)$ and $\xi^{\prime} \in \Sigma(r) \cup \Gamma_{\mathrm{nr}, \mathrm{ch}}(H, r)$ such that $\xi \equiv \xi^{\prime}$. This condition can be decided efficiently, as (i) there are at most $O\left(|V(H)|^{k+1}\right)$ iso-quadruples of $H$ relative to $r$ that have to be tested, (ii) $\left|\Sigma(r) \cup \Gamma_{\mathrm{nr}, \mathrm{ch}}(H, r)\right|$ is bounded by a polynomial of the combined size of the input and the set of frequent patterns computed before $H$, and (iii) equivalence between iso-quadruples can be decided in polynomial time (see the remarks before this paragraph). 


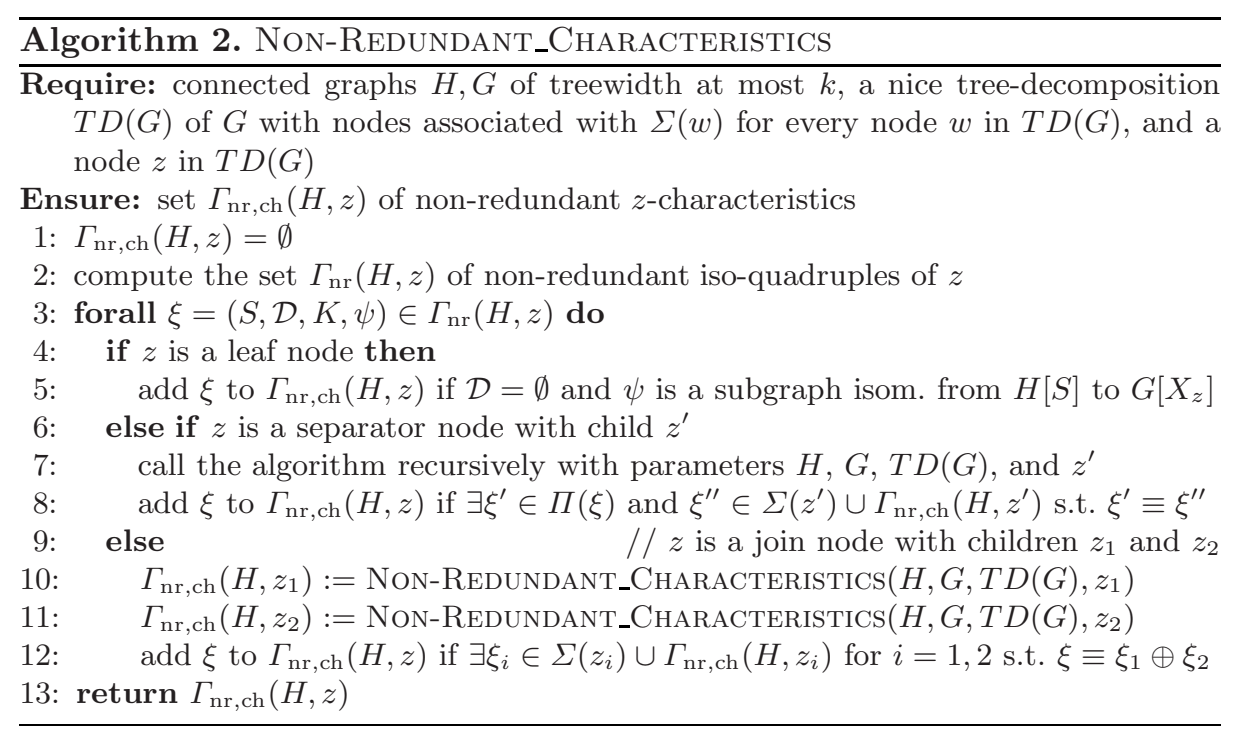

Thus, we only have to discuss the computation of the $\Gamma_{\mathrm{nr}, \mathrm{ch}}(H, z)$ 's for the nodes in $T D(G)$. Before going into the details, we first note that if $H$ is frequent then $\Gamma_{\mathrm{nr}, \mathrm{ch}}(H, z)$ must be added to $\Sigma(z)$ after the frequency counting step in Algorithm 1. A recursive algorithm computing $\Gamma_{\mathrm{nr}, \mathrm{ch}}(H, z)$ for every node $z$ in $T D(G)$ is given in Algorithm 2 For the current node $z$ in $T D(G)$, the algorithm first sets $\Gamma_{\mathrm{nr}, \mathrm{ch}}(H, z)$ to $\emptyset$ and then computes the set $\Gamma_{\mathrm{nr}}(H, z)$ of non-redundant iso-quadruples relative to $z$. As shown earlier, $\Gamma_{\mathrm{nr}}(H, z)$ can be computed in time polynomial in the combined size of $H$ and $G$, as they have bounded treewidth. Then, depending on the type of $z$, for every iso-quadruple $\xi=(S, \mathcal{D}, K, \psi)$ in $\Gamma_{\mathrm{nr}}(H, z)$, one of the following steps is performed:

$z$ is a Leaf Node: We can apply Lemma 2 for this case, but only for the elements in $\Gamma_{\mathrm{nr}}(H, z)$.

$z$ is a Separator Node: The proof of the lemma stated below for this case follows directly from Proposition 1 and Lemma 3 . We recall that $\Pi(\xi)$ denotes the set of iso-quadruples in $\Gamma(H, z)$ satisfying the conditions before Lemma 3.

Lemma 6. Let $H$ be a strong candidate pattern generated by levelwise search, $z$ be a separator node in $T D(G), z^{\prime}$ be the child of $z$, and $\xi \in \Gamma_{\mathrm{nr}}(H, z)$. Then $\xi$ is a $z$-characteristic iff there exist $\xi^{\prime} \in \Pi(\xi)$ and $\xi^{\prime \prime} \in \Sigma\left(z^{\prime}\right) \cup \Gamma_{\mathrm{nr}, \mathrm{ch}}\left(H, z^{\prime}\right)$ such that $\xi^{\prime} \equiv \xi^{\prime \prime}$.

Since $\Pi(\xi)$ has at most $(k+1) ! \cdot|V(H)|^{k+1}$ elements and it can be computed efficiently, we can decide in polynomial time whether it has an element equivalent to an element of $\Sigma\left(z^{\prime}\right) \cup \Gamma_{\mathrm{nr}, \mathrm{ch}}\left(H, z^{\prime}\right)$, that is, whether $\xi$ is a $z$-characteristic. Combining this result with Lemma 5 , we have that $\Gamma_{\mathrm{nr}, \mathrm{ch}}(H, z)$ can be computed in time polynomial in the size of $H$. 
$z$ is a Join Node: Let $z_{1}$ and $z_{2}$ be the children of $z$ and let $\xi_{1}=\left(S_{1}, \mathcal{D}_{1}, K_{1}\right.$, $\left.\psi_{1}\right)$ and $\xi_{2}=\left(S_{2}, \mathcal{D}_{2}, K_{2}, \psi_{2}\right)$ be $z_{1}$ - and $z_{2}$-characteristics, respectively. We may assume w.l.o.g. that $K_{1}$ and $K_{2}$ are vertex disjoint. Then the join of $\xi_{1}$ and $\xi_{2}$, denoted $\xi_{1} \oplus \xi_{2}$, is an iso-quadruple $\xi=(S, \mathcal{D}, K, \psi)$, where

$-S=\left\{w_{u}: u \in \psi_{1}\left(S_{1}\right) \cup \psi_{2}\left(S_{2}\right)\right\}$ is a set of new vertices,

$-\mathcal{D}=\mathcal{D}_{1} \cup \mathcal{D}_{2}$,

- $K$ is the graph obtained by adding to the set $\mathcal{D}$ of connected components (i) the set $S$ of vertices, (ii) the edge $\left\{w_{\psi_{i}(u)}, w_{\psi_{i}(v)}\right\}$ for all $u, v \in S_{i}$ such that $\{u, v\} \in E\left(K_{i}\right)$, and (iii) the edge $\left\{w_{\psi(u)}, v\right\}$ for every $u \in S_{i}$ and $v \in V\left(\mathcal{D}_{i}\right)$ such that $\{u, v\} \in E\left(K_{i}\right)$ for $i=1,2$, and

$-\psi: S \rightarrow X_{z}$ is defined by $\psi\left(w_{u}\right)=u$ for every $w_{u} \in S$.

Notice that $|S| \leq k+1$ because $\psi_{1}\left(S_{1}\right) \cup \psi_{2}\left(S_{2}\right) \subseteq X_{z_{1}} \cup X_{z_{2}}=X_{z}$ and $\left|X_{z}\right| \leq$ $k+1$, as $z$ is a join node. Using the above definition, we give a lemma characterizing the set of $z$-characteristics of a candidate pattern $H$ in terms of $z_{1}$ and $z_{2}$-characteristics computed before $H$. Although the lemma is used only for non-redundant iso-quadruples, we formulate it in a general form. The following lemma follows from Proposition 1 and Lemma 4

Lemma 7. Let $H$ be a strong candidate pattern generated by levelwise search, $z$ be a join node in $T D(G)$, and $z_{1}$ and $z_{2}$ be the children of $z$. Let $\xi \in \Gamma(H, z)$. Then $\xi$ is a $z$-characteristic iff there exist $\xi_{1} \in \Sigma\left(z_{1}\right) \cup \Gamma_{\mathrm{nr}, \mathrm{ch}}\left(H, z_{1}\right)$ and $\xi_{2} \in$ $\Sigma\left(z_{2}\right) \cup \Gamma_{\mathrm{nr}, \mathrm{ch}}\left(H, z_{2}\right)$ such that $\xi_{1} \oplus \xi_{2} \equiv \xi$.

Using the arguments applied to separator nodes, we have that $\Gamma_{\mathrm{nr}, \mathrm{ch}}(H, z)$ can be computed in time polynomial in the combined size of $H$ and the set of frequent patterns computed before $H$; the second term follows from the facts that (i) the number of pairs $\left(\xi_{1}, \xi_{2}\right)$ for which the join must be computed and tested is at most $\left|\Sigma\left(z_{1}\right) \cup \Gamma_{\mathrm{nr}, \mathrm{ch}}\left(H, z_{1}\right)\right| \cdot\left|\Sigma\left(z_{2}\right) \cup \Gamma_{\mathrm{nr}, \mathrm{ch}}\left(H, z_{2}\right)\right|$, and (ii) for $i=1,2$, $\left|\Sigma\left(z_{i}\right) \cup \Gamma_{\mathrm{nr}, \mathrm{ch}}\left(H, z_{i}\right)\right|$ is bounded by the combined size of $H$ and the set of frequent patterns computed before $H$. Putting together Lemmas [6] and 7 with the remarks above, we have the following lemma:

Lemma 8. For every strong candidate pattern $H$ generated by levelwise search and for every $G$ in $D B$, both of bounded treewidth, Algorithm 2 is correct and computes $\Gamma_{\mathrm{nr}, \mathrm{ch}}(H, z)$ for every node $z$ in $T D(G)$ in time polynomial in the combined size of $H, G$, and the set of frequent patterns computed before $H$.

The following theorem states that subgraph isomorphism between graphs of bounded treewidth can be decided in incremental polynomial time.

Theorem 3. Let $D B$ be a database of transaction graphs of treewidth at most $k$ and $H$ be a strong candidate pattern of treewidth at most $k$ which is generated by a levelwise search algorithm mining frequent connected subgraphs from $D B$. Let $T D(G)$ be some fixed nice tree-decomposition used by the mining algorithm and let $r$ be the root of $T D(G)$. Then $H \leq G$ iff

$$
\exists(S, \mathcal{D}, H, \psi) \in \Gamma(H, r) \text { equivalent to some } \xi \in \Sigma(r) \cup \Gamma_{\mathrm{nr}, \mathrm{ch}}(H, r) .
$$

Furthermore, condition (11) can be decided in time polynomial in the combined size of $H, G$, and the set of frequent patterns computed before $H$. 


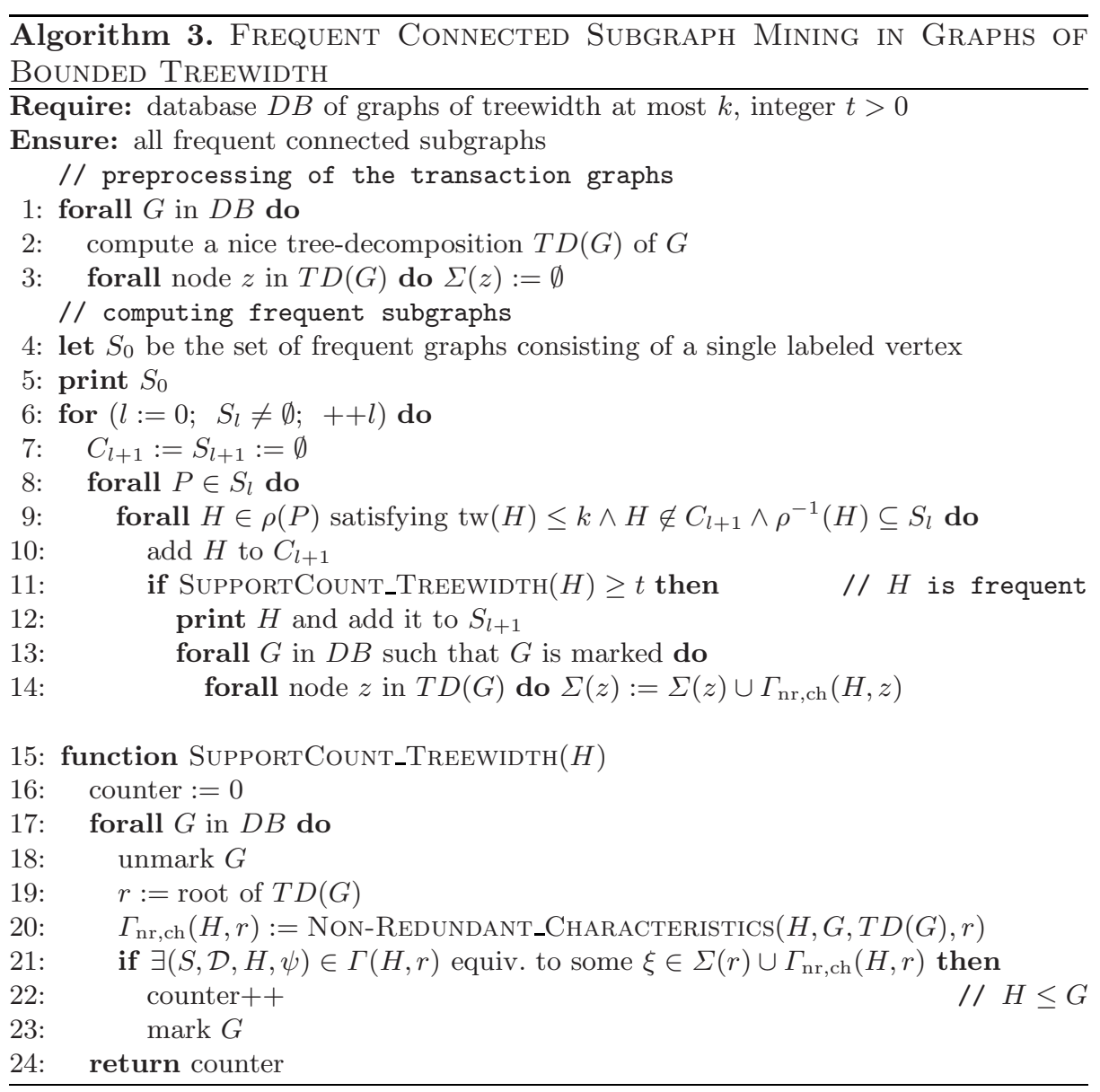

Proof. The first part of the theorem holds by Lemma 1 and Proposition 1 Regarding the second, i.e., complexity part, we first note that by Lemma 8 , $\Gamma_{\mathrm{nr}, \mathrm{ch}}(H, r)$ can be computed in time polynomial in the combined size of $H, G$, and the set of frequent patterns computed before $H$. Furthermore, the number of iso-quadruples in $\Gamma(H, r)$ to be checked in (1) is bounded by $(k+1) ! \cdot\left|V(H)^{k+1}\right|$. The statement then follows by noting that $\left|\Sigma(r) \cup \Gamma_{\mathrm{nr}, \mathrm{ch}}(H, r)\right|$ is bounded by a polynomial of the combined size of $H$ and the set of frequent patterns listed before $H$ and that it can be decided in time linear in the size of $H$ whether an iso-quadruple $\xi \in \Gamma(H, r)$ is an element of $\Sigma(r) \cup \Gamma_{\mathrm{nr}, \mathrm{ch}}(H, r)$.

\subsection{The Mining Algorithm}

Combining the results of the previous sections, we now show that the FCSM problem can be solved in incremental polynomial time from graphs of bounded treewidth. A listing algorithm solving this problem is given in Algorithm 3 . 
In the preprocessing step (lines 1-3 of Algorithm 3), we first compute a nice tree decomposition $T D(G)$ for every transaction graph $G$ and initialize the set variables $\Sigma(z)$ for every node $z$ in $T D(G)$. Since a nice tree-decomposition of treewidth $k$ can be computed in linear time for graphs of treewidth at most $k[3$, the preprocessing step can be performed in time linear in the size of $D B$.

Lines 4-14, the main part of Algorithm 3, is an adaptation of the generic levelwise search Algorithm 11. One of the differences is that in lines 13-14 we have to update the sets of non-redundant characteristics for frequent patterns. Since $\mathcal{G}$ in Algorithm 1 is now the class of graphs of treewidth at most $k$, deciding whether $H$ is an element of $\mathcal{G}$ corresponds to checking whether $\operatorname{tw}(H) \leq k$ (see line 5 and line 9 in Algorithms 1 and 3, respectively). As mentioned above, for constant $k$, this can be decided in linear time 3. Since the sizes of $\rho(H)$ and $\rho^{-1}(H)$ are both bounded by the size of $D B$, the size of $C_{l+1}$ is bounded by a polynomial of the combined size of the set of frequent patterns computed before $H$, and isomorphism between graphs of bounded treewidth can be decided in polynomial time [2], the conditions in line 9 of Algorithm 3 can be checked in time polynomial in the combined size of $D B$ and the set of frequent patterns listed before $H$. Finally, by Theorem 3. the subgraph isomorphism (lines 20-21) can be decided in time polynomial in the combined size of $H, G$, and the set of frequent patterns computed before $H$. Putting all these together, we get the main result of this paper:

Theorem 4. For graphs of bounded treewidth, the FCSM problem can be solved in incremental polynomial time.

\section{Concluding Remarks}

The main result of this paper is formulated in Theorem 4 above. There are only a few results concerning the complexity of the FCSM problem. In particular, for arbitrary transaction graphs, this problem cannot be solved in output-polynomial time, unless $P=N P$ [10]. If, however, the transaction graphs are restricted to trees then it can be solved in incremental polynomial time 511]. The positive result on trees is generalized to outerplanar graphs in 11. However, this result is shown for a constrained subgraph isomorphism, called BBP subgraph isomorphism, that maps biconnected components to biconnected components and bridges to bridges. Since trees have no biconnected components, BBP subgraph isomorphism is equivalent to subgraph isomorphism between trees. As far as we know, no non-trivial tractable graph classes beyond trees have so far been identified for FCSM problem.

Since subgraph isomorphism remains NP-complete for connected graphs of bounded treewidth, our result provides an example of a frequent pattern mining problem, when the matching operator is NP-hard, but efficient mining is still possible. To the best of our knowledge, existing pattern mining algorithms are all resorted to problems with tractable matching operators. Our result shows that efficient pattern mining is possible even for NP-hard matching operators. 
Deriving and implementing a practical algorithm from the algorithm described in this paper is an interesting task for future work. This plan is motivated by practical applications, e.g., in computational drug discovery where molecular graphs mostly have treewidth at most 3 .

We close the paper with an interesting problem: Can the FCSM problem be solved with polynomial delay for graphs of bounded treewidth?

Acknowledgements. We thank one of the anonymous reviewers, Mario Boley, and Kristian Kersting for useful comments on improving the presentation. Tamás Horváth was partially supported by the German Federal Ministry of Economy and Technology under the Theseus Project. He thanks György Turán and Stefan Wrobel for interesting discussions on frequent subgraph mining. Jan Ramon is a post-doctoral fellow of the Fund for Scientific Research of Flanders (FWOVlaanderen).

\section{References}

1. Arnborg, S., Proskurowski, A.: Linear time algorithms for NP-hard problems on graphs embedded in $k$-trees. Discrete Applied Mathematics 23, 11-24 (1989)

2. Bodlaender, H.L.: A tourist guide through treewidth. Acta Cybernetica 11(1-2), 1-22 (1993)

3. Bodlaender, H.L.: A linear-time algorithm for finding tree-decompositions of small treewidth. SIAM Journal on Computing 25(6), 1305-1317 (1996)

4. Bodlaender, H.L.: A partial $k$-arboretum of graphs with bounded treewidth. Theoretical Computer Science 209(1-2), 1-45 (1998)

5. Chi, Y., Nijssen, S., Muntz, R.R., Kok, J.N.: Frequent subtree mining - an overview. Fundamenta Informaticae 66, 161-198 (2005)

6. Deshpande, M., Kuramochi, M., Wale, N., Karypis, G.: Frequent substructurebased approaches for classifying chemical compounds. IEEE Transactions on Knowledge and Data Engineering 17(8), 1036-1050 (2005)

7. Garey, M.R., Johnson, D.S.: Computers and Intractability: A Guide to the Theory of NP-completeness. Freeman, San Francisco (1979)

8. Gupta, A., Nishimura, N.: The complexity of subgraph isomorphism for classes of partial k-trees. Theoretical Computer Science 164(1\&2), 287-298 (1996)

9. Hajiaghayi, M.T., Nishimura, N.: Subgraph isomorphism, log-bounded fragmentation, and graphs of (locally) bounded treewidth. Journal of Computer and System Sciences 73(5), 755-768 (2007)

10. Horváth, T., Bringmann, B., De Raedt, L.: Frequent hypergraph mining. In: Muggleton, S., Otero, R., Tamaddoni-Nezhad, A. (eds.) ILP 2006. LNCS (LNAI), vol. 4455, pp. 244-259. Springer, Heidelberg (2007)

11. Horváth, T., Ramon, J., Wrobel, S.: Frequent subgraph mining in outerplanar graphs. In: Proc. of the 12th ACM SIGKDD Int. Conf. on Knowledge Discovery and Data Mining, pp. 197-206. ACP Press, New York (2006)

12. Johnson, D.S., Papadimitriou, C.H., Yannakakis, M.: On generating all maximal independent sets. Information Processing Letters 27(3), 119-123 (1988)

13. Matoušek, J., Thomas, R.: On the complexity of finding iso- and other morphisms for partial k-trees. Discrete Mathematics 108(1-3), 343-364 (1992)

14. Robertson, N., Seymour, P.D.: Graph minors. II. Algorithmic aspects of treewidth. Journal of Algorithms 7(3), 309-322 (1986) 